\title{
The Two Worlds of John Keats -An Analysis of Life and Death in To Autumn
}

\author{
Chen $\mathrm{Yao}^{1, *}$ \\ Department of English, Tianjin University of Finance and Economics, Tianjin, China \\ *18731682964@163.com
}

\begin{abstract}
John Keats, a genius poet, is like a sparkling morning dew that disappears swiftly when the rising sun begins to give warmth on earth. His poems attract people not only because of its aesthetic beauty but also because of the profound thinking on life and death they reflect. Keats's poems present his complex emotion of life and death. When he is tired of the reality, he expresses his negative attitudes towards life in his poems; when he is in positive attitudes, his poems praise the truth and the beauty in life. This thesis mainly focuses on the two worlds in his To Autumn from two aspects for the interpretation of the view towards life and death. Keats's poetry illustrates negative and positive attitudes towards life and death and To Autumn not only conveys his treasure of life but also express his reconciliation to and tolerance for death.
\end{abstract}

Keywords: John Keats; To Autumn; Positive and Negative Attitudes; View of Life and Death

\section{约翰・济慈的两个世界 \\ 《秋颂》之生死观分析}

陈瑶 ${ }^{1, a}$

天津财经大学英语系, 天津, 中国

18731682964@163.com

\section{摘要}

天才诗人约翰 - 济慈, 就像一朵闪闪发光的晨露, 当冉舟升起的太阳开始给地球带来温暖时, 它迅速 消失。他的诗之所以吸引人, 不仅是因为它的美学价值, 更是因为它所反映的对生死观的深刻思考。 济慈的诗歌表现了他复杂的生死情感。当他对现实感到厌倦时, 他在诗歌中表现出对生活的消极态度; 当他处于积极态度时, 他的诗歌赞美生活的真与美。本文主要从两个方面对《秋颂》中的两个世界进 行解读。《秋颂》中济慈所体现的对生死观的消极态度和积极态度, 不仅传达了他对生命的珍视, 而且 表达了他对死亡的和解与宽容。

关键词: 约翰・济慈; 《秋颂》; 积极与消极态度; 生死观

\section{1. 简介}

约翰 - 济慈是 18、19 世纪英国文学中最杰出的浪 漫主义诗人之一, 共发表了五十四首诗。在他发展的每 一个阶段, 都在挑战各种各样的诗歌形式, 从十四行诗 到斯宾塞罗曼史诗, 再到弥尔顿史诗, 以自己独特能量 的融合, 对冲突的观点和力量的控制, 偶尔干涩的讽刺 机智和诗意的自我意识, 重新定义了它们的可能性。以 英国颂歌为例, 济慈发表于 1819 年的五首 “伟大的颂 歌”是对它的完美定义 ${ }^{[1]}$ 。

《秋颂》是济慈于 1819 年 9 月在温彻斯特写的一 首著名的颂歌。这不仅是他最后一部作品, 也是他最成
熟、最令人满意的一首颂诗。深受评论家和诗歌爱好者 的喜爱, 并被认定为一部完美的作品。贝特对其给予了 高度尊重, 说一代又一代的读者 “发现它是英语中最完 美的诗歌之一” ${ }^{[2]}$ 。《秋颂》是诗人最简单的一首颂诗, 无论从形式上还是描写上都是如此。在他对秋天的赞歌 中, 没有任何令人困惑或复杂的东西: 它的果实, 它的 花朵, 它的燕子为迁徙而聚集的歌声等等。这首诗的非 凡成就在于它能够探索和发展丰富的主题, 而不会扰乱 它对秋天平静、柔和和可爱的描写。

文章刚开始的部分是对济慈悲惨人生经历的展开, 这被认为是济慈对生死持否定态度的根源。之后, 通过 对济慈脱离现实生活的论述, 阐述了济慈逐渐成熟的人 生态度和超越性的死亡观。最后, 以济慈的《秋颂》为 
例，从其消极与积极两个世界中进一步探讨济慈对生命 的欣赏和对死亡的平和态度。

\section{2. 济慈生死观的形成}

人的生命随时可能结束, 活在这个世界上便意味着 走向死亡。生命是短暂的, 死亡将终结一切, 包括痛苦。 因此, 生命是值得珍惜的, 沉溺于自我的苦难是对生命 的浪费。

\section{1 对生死观的消极态度}

冊庸置疑, 作家的个人经历和社会背景或多或少地 影响着他的作品和对生死的态度。对济慈作为一个诗人 的每一次批判性评价都必须从接受他的作品和他的生 活是不可分割的这一事实开始 ${ }^{[3]}$ 。因此, 研究他的生死 观，人们首先要了解他的生平并掌握大概的背景知识。 背景是非常复杂的, 它们深刻地影响着诗人, 使他形成 了独特的视角, 促进了他文学艺术的进步。

\section{1.1 文化背景}

如果人们把济慈和他的文化背景联系起来，就会更 容易理解济慈的生死观, 如将他和他的诗歌放在他所处 的特别是浪漫主义和艺术革命运动的时代背景下。

在工业革命、法国大革命和自由、平等、博爱思想 的影响下，浪漫主义运动是十八世纪末经济、政治和意 识形态剧变的产物。在这一时期, 济慈的诗歌被认为是 更个人的，虽然有时因为他与伦敦学派的积极分子如雪 莱的密切关系而不可避免的带有政治性 ${ }^{[4]}$ 。浪漫主义者 虽然在很多方面都是不同的, 但也存在很多共同点。浪 漫主义者作品的一个普遍特点是对资产阶级社会的不 满。伟大的浪漫主义诗歌是诗人对革命的幻灭或在绝望 的心情下写成的。由于这种绝望, 他们的作品或多或少 带有一种逃避现实的色彩, 而且这些浪漫主义者在描绘 人的精神和情感生活时, 主要依靠想象。济慈敏锐地意 识到外在的、日常的表象世界与内在的、深奥的、不朽 的善与美的真理之间的鸿沟, 这是一个只有通过想象才 能进入的领域。

\subsection{2 个人经历}

为了全面而真实地了解济慈, 人们不得不提及他的 个人经历。他的每一首诗都源于他的实际经验。济慈在 短暂的一生中经历了各种不寻常的艰难挫折一一父母 早逝, 文学界的严厉批评, 与范妮・布朗的无果之爱, 以及他致命的疾病。显而易见，他的生活充满了痛苦， 死亡是贯穿他诗歌各个方面的永恒主题。

1819 年 3 月, 济慈在写给乔治和乔治娜的信中提 到: “这就是世界。因此, 我们不能指望拥有更多去享
乐的时间……当我们在笑的时候，一些麻烦的种子便被 放入了广衰的可耕地中” ${ }^{[5]}$ 。这封信生动地揭露了济慈 对世界的失望。他觉得快乐的时光总是短暂的且所有的 快乐都会被毁灭, 人类没有能力保持美丽和快乐的事 物。

济慈的人生经历导致了他的痛苦感受。他看到别人 的死亡, 深受创伤, 并引起了他对死亡的恐慌。他认为 他所拥有的一切注定都要失去。面对无处不在的死亡, 人是脆弱而无力的。济慈根深蒂固的死亡观念暗示了他 对生命存在的不安全感。

\section{2 对生死观的积极态度}

济慈的诗歌中也体现了他对生死的积极态度。济慈 生前被病魔和死亡包围, 所以他对死亡很熟悉, 这使他 面对死亡时能够保持平静的态度。其次, 由于济慈多次 遭受各种苦难，所以他对现实世界并没有情感上的依 恋。因此, 济慈的诗歌揭示了平静的生活情调。他在信 中深刻地表达了他对生与死的积极态度，他写道: “人 的状态并不比玫瑰好多少。它在一个美丽的早晨绽放, 自得其乐。但它逃不掉随之而来的寒风与烈日，它被摧 毁了。它们与世界本身一样是土生土长的” ${ }^{[5]}$ 。在济慈 眼中, 人的生存和死亡与自然的变迁是一样的。因此, 济慈既不沉溺于世俗生活, 也不惧怕死亡。这种冷静、 积极的生死观赋予了济慈诗歌一种超然感。

\subsection{1 对生与死的超然之感}

济慈对生死的冷静态度, 源于他对死亡的熟悉。死 亡夺走了他生命中所珍视的东西。因此, 济慈认为生命 中任何美好和快乐的事物都不可能在世界上永恒存在。 此外, 济慈还相信人类可以通过死亡来摆脱痛苦和疾 病。因此, 他没有生活在对死亡的否认中。这种冷静的 态度使他的诗歌传达出一种超然之感。

生与死是分不开的。济慈接受了死亡永远存在的事 实, 并敞开心扉, 而不是沉溺于悲伤之中。生命是短暂 的, 生活在死亡的阴影下是不明智的。除此之外, 生命 由每一个活着的时刻组成，每一天都充满了神秘和发 现, 这给诗人带来了不同的生活感受 ${ }^{[6]}$ 。因此, 济慈不 再沉溺于丧亲之痛, 平静地接受了生命中所失去的部 分。他开阔了视野, 注重生活的变化。在广阔的视野中, 济慈获得了一种超然的感觉。人的生命与世界息息相 关, 人的生命意识被世界唤醒。放纵自己是不幸的祸根, 多样性是生命的宝藏。济慈以开放的心态, 实现了内心 的平静。

\subsection{2 向死而生: 对生命的赞扬与珍视}

死亡是不可避免的, 但人们可以正面面对死亡。济 慈呼吁人们对生活保持积极的态度, 热情地参与社会活 动是济慈的主要观点。在他的诗歌中, 与审美形式的斗 
争变成了对经验极限的意义斗争, 他的艺术形式似乎体 现和解释了死亡和欲望的冲突。由于济慈对美的强烈热 爱和悲惨的短暂人生, 这首诗一直在向读者显示一种紧 迫感 ${ }^{[7]}$ 。济慈对经验、想象、艺术和幻觉之间的关系进 行了深入的思考, 既没有感情用事, 也没有愤世痴俗, 而是用微妙的美和常常令人惊讶的方式以揭示真理为 乐。

当人们阅读济慈的诗歌时, 人们便可以洞察他的内 心世界, 并发现诗人意识到了现实中缺乏的美和与可怕 命运抗争的力量。同时, 诗人明白死亡与自然生命的死 亡和社会事物的更替并无太大区别。死亡是人的意志所 不能改变的自然规律。《秋颂》是以一种恬静愉悦的语 调写成的, 人们也能感受到济慈是多么珍惜字里行间的 快乐。

\section{3. 《秋颂》中的两个世界}

人们很容易在《秋颂》中找到两个世界: 消极世界 和积极世界。《秋颂》, 以其具体的图像目录, 最密切地 描述了一个在地球上实现的天堂, 同时也关注与季节相 关的原型符号。在这首诗中, 秋天从开始的成长、成熟, 到最后死亡的临近, 理想和现实之间有一个完美的结 合。在《秋颂》中, 济慈本人运用丰富的想象力将两个 领域结合起来, 通过创造一个完全不同于现实的理想世 界, 揭示了他对永恒美的追求。在这首诗中, 济慈的生 死观强调生命的有限性。他传达了生活在这个世界上的 人们随时都面临死亡的威胁。然而, 和平的生活状态可 以通过珍惜生命的瞬间和平静地面对死亡来实现。生命 是由每一刻组成的, 因此珍惜生命中的每一刻就是对生 活的尊重。

\section{1 《秋颂》中的消极世界}

关于这首颂诗的主题, 自它第一次出现以来, 学者 们便提出了许多不同的看法。一些评论家把这首诗与诗 人的生活联系起来, 得出了不同的见解。达戈尔说, “《秋 颂》是自然界的一个季节的结束, 也是他生命的季节, 它是自然界成就的总结, 同时也是一种半意识的告别生 命的姿态” ${ }^{[8]}$ 。秋天本身就是一年中的一个回顾时间。 《秋颂》中, 济慈回首自己的充实生活, 感受自然生命 周期完成的喜悦。尽管从外表上看, 整首诗充满了欢乐, 但忧郁却潜伏在黑暗中, 因为像秋天这样美丽的事物一 定会因为时间的流逝而消逝。

这首诗有三节, 每节由十一行组成。在第一节中, 诗人描述了一幅秋天的丰收景象, 向人们展示了秋天的 自然成果: 成熟的果实、胀大的葫芦、羽状的榛子、盛 开的的鲜花、翩翩起舞的蜜蜂和令人愉快的温暖日子。 这样的描述让人们脑海中浮现出这样一个画面: 秋天正 是盛年, 万物成熟, 准备丰收。然而, 在第二节中, “秋 天” 是 “随意地坐在打麦场上” 或 “倒卧在收割一半的 田垄旁” ${ }^{[9]}$, 诗人向人们描绘了一幅栩栩如生的画面,
人们在田里忙着收割。秋天的收获是令人满意的: 粮仓 里装满了粮食, 在微风的轻拂下, 半收割的犁沟、麦田、 缠绕的花朵和清澈的小溪都浸泡在罂粟的烟雾中。但他 为什么不采取行动收割呢? 因为收割庄稼的人耽误了, 他太清楚秋天美景的消失即短暂的秋天将导致收割的 失败, 因此他不采取任何行动。

当来到最后一节的时候, 秋天就快结束了, 冬天来 临的暗示也清晰可见。在诗人的笔下，所有的生物都蜕 变成了秋天的美妙合唱, 唱出了最丰富最精彩的歌曲:

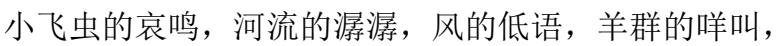
还有篱下蟋蟀的歌唱, 红胸知更鸟的呼啸和从飞燕子的 呢喃。多彩的秋歌丰富了它的成熟之美, 但瞬间被唤起 了失落的感觉。在最后一行, 现在时态动词给人一种强 烈的存在感，它把过去聚集起来，并被推向未来: “红 色胸脯的知更鸟从花园里呼啸而出, 从飞的燕子在空中 吹吹喳喳” [9]。在济慈的颂诗中, 强烈的体验和神话的 视野第一次在纯自然的环境中达到了平衡。这一节起到 了一个过渡功能, 它改变了从夏天到冬天的季节, 鸟类 从停留到离开，生命从振奋到疲崽。“成年的羔羊” 形 象是生命流逝的暗示, 因为羔羊在春天是幼小的, 直到 秋天才长大。“燕子” 是指停留和离开的界限。燕子是 夏天的鸟, 当它离开时, 冬天就来了。

济慈被美丽的风景感动, 但同时也在感叹人类生命 的短暂。因此, 在享受了美丽的景色和丰收的喜悦之后, 一种忧愁感弥漫开来。因为他预见到, 在金秋的背后, 是一个没有生命力的冬天。到那时, 树上没有叶子, 地 上没有植物, 黯淡的形象会造成痛苦的感觉。这种特殊 的诗意表达与济慈的人生经历和人生信念有关。在济慈 的诗歌中, 死亡的阴影总是在他的脑海中游荡, 生命的 短暂在他的诗歌中传播。

虽然这是一首关于秋天的颂歌，但也提到了夏天和 即将到来的冬天。时间在这首诗中永不停歇, 读者从头 到尾都能感受到时间的流逝。然而, 人们不应该永远被 这首看似欢乐的诗所欺骗。即将来临的失落感弥漫在诗 中, 悲伤是季节创造力的基础, 白昼将失, 飞虫、羔羊、 蟋蟀和小鸟似乎都意识到即将来临的黑暗。押韵模式也 表达了忧郁的感觉, 并进一步暗示了死亡的主题。正如 稍纵即逝的感觉从未离开济慈, 死亡的主题从未真正离 开过这首诗。

\section{2 《秋颂》中的积极世界}

《秋颂》是以一种相当欢快的语调写成的，人们也 能感受到济慈是多么珍惜字里行间的幸福。济慈在《秋 颂》中表达了积极态度和平和的生活状态: 珍惜生命中 的每一刻, 平静地面对死亡, 才能达到平和的生命状态。 在这首诗中, 诗人完全接受了自然世界, 它是成熟、充 实、消逝和死亡的混合体。诗人被秋天的美丽果实深深 震撼, 因而写下了这首伟大的颂诗。然而, 他写这首颂 诗不仅是因为他被感动了，因而有把自己的幸福写下来 的冲动, 还因为他想让快乐的感觉和美丽的景色永远留 在他的潜意识里。 
尽管冬天即将来临, 但深秋的暖意却给诗人带来了 多彩的欢庆之美：第一节描述了色彩纷呈的 “秋色”, 第二节则是对百忙之中偷得片刻清闲的 “秋人” 描写, 第三节展现了自然生物栖息地中的 “秋声”，诗人能够 以真诚且有意义的方式体验这些美丽 ${ }^{[10]}$ 。从热爱现实的 角度看, 这首诗充满了乐观情怀, 他对美的追求和对美 感的歌颂是具体而真实的, 将词与美的关系很好地联系 在一起。

这首诗的第一节代表了秋天, 它涉及到自然过程的 促进、生长和最终成熟, 同时又给人留下了季节不会结 束的印象。在这一节中, 果实仍在成熟, 花蕾仍在温暖 的天气中开放。在第二节中, 秋天被拟人化为一个收割 庄稼的人, 读者可以看到它以各种各样的姿态执行着为 来年提供食物所必需的劳动任务。虽然缺乏明确的行 动, 但所有的行动都是温和的。这首诗中的最后一节代 表着被死亡束缚的生命态度。秋天是一个边界, 因为它 把充满活力的季节转移到毫无生气的冬天。济慈并不为 失去春天和夏天而悲伤。相反, 他心胸开阔, 时刻拥抱 秋天的到来。尽管秋天的音乐是 “悲歌”，济慈仍然珍 视秋天的景色。“不要想他们, 你也有你的音乐” ${ }^{[9]}$ 这句 话表达了济慈对生命的珍惜。除此之外, “将逝的一天” 和 “残梗散碎的田野” 都是代表被死亡束缚的生命意象。 然而, 济慈用一种平和的心态描绘了他的死亡观念。“将 逝的一天” 在美丽的夕阳余晖中结束, 波状的云把 “残 梗散碎的田野” 染成玫瑰色。虽然这一天即将结束, 平 原上的植物也将枯萎, 但现存的景色值得赞扬。在这里, 济慈揭示了他的人生智慧。他珍惜现在的生活, 在短暂 的生命中享受美好快乐的时刻。同时, 被死亡束缚的生 命态度贯穿于诗歌之中, 它揭示了济慈对死亡平和的态 度。当生命中快乐的时刻出现时, 他满怀激情地享受着; 当死亡降临时, 他平静地接受它。

此外, 济慈在这首诗的第一行通过相反阶段的意象 来表达他的辩证人生观。它们是 “迷雾” 和 “累累的果 实”, “知已” 和 “密谋”, “负荷” 和 “祝福” [9]。所有 这些相反的意象都传达了诗人无法解释的情感一诗人 既喜又悲。当他陶醉于眼前的欢乐景色时, 一种失落感 刺痛了他的心。就像人类一样, 自然也有生命周期, 现 在所有美好的事物将来都会毁灭。从另一个角度看, 这 种快乐的描绘表达了济慈对生命的热爱。他珍视世界上 所有美丽的事物, 特别是大自然的奇景。诗人屈从于秋 天的仙境, 忘记了痛苦。

总之，这首诗表达了诗人对生活中美好事物的欣 赏。济慈以广阔的视野看待人生, 他珍视世界之美, 享 受幸福; 同时, 他也持悲观的观点, 认为现在的一切都 是短暂的, 注定要走向灭亡。因此, 诗人认为沉溺于幸 福或陷入忧郁都是不明智的, 辩证地看待生死才是一种 明智的人生态度。

\section{4. 结语}

本文分析了济慈生死观的消极态度和积极态度。这 两个世界构成了济慈的一生。本文以济慈的《秋颂》为
例，进一步探讨济慈对生命的鉴赏和对死亡的平和态 度。他既不对失去的时间感到懊悔, 也不担心即将到来 的死亡。相反, 他珍惜现在。济慈在这首诗中传达了一 种超然的生死观, 给后人以启示。

济慈逃离现实生活, 生活在他的想象世界里。济慈 诗歌中的死亡观不是为死亡服务, 而是为生命服务。生 命是短暂的, 所以活着的时刻是宝贵的。此外, 他面对 生活的勇气值得钦佩。济慈选择开阔视野, 勇于发现世 界上美丽的事物, 辩证的生死观有助于他对面对生死时 的冷静和超然。当他享受世界之美时, 被死亡束缚的生 活态度起着自我控制的作用，阻止他自我吸收; 当他面 对死亡时，被死亡束缚的生活态度是一种安慰，抚慰他 的痛苦, 带给他一个平和的心情。他的诗歌给读者以平 静的生死观, 呼吁读者珍惜当下的生命, 坦然接受必然 的死亡。

因此, 本文认为济慈的生死发展观培养了后人的生 命态度。他对生活态度和思想的转变传达了他的生活信 仰和理念。总之, 他的消极态度和忧郁使读者产生了共 鸣, 他的积极态度和自我解放激励着人们探索一条摆脱 伤悲的道路, 他的同情心和人文关怀给人们带来生命希 望的同时，激起了人们对生命的珍视感。

\section{REFERENCES}

[1] Bennett, A. (1994) Keats, Narrative and Audience. Cambridge University Press, Cambridge.

[2] Bate, J. (1964) Keats:A collection of Critical Essays. Belknap Press, Cambridge.

[3] Harrison, P. (2003) The Dominion of the Dead. Chicago University Press, Chicago.

[4] Abram, M. (1979) The Norton Anthology of English Literature Volume 2. W.W. Norton Company, London.

[5] Keats, J. (1958) The Letters of John Keats. Harvard University Press, Harvard.

[6] Clemens, J. (2003) The Romanticism of Contemporary Theory: Institution, Aesthetics, Nihilism. Ashgate, Aldershot.

[7] Vendler, H. (2014) The Odes of John Keats. Harvard University Press, Cambridge.

[8] Dagorn, L. (2016) John Keats, The Final Season in a Young Poet's Life and His Final Poem. Cambridge University Press, Cambridge.

[9] Keats, J. (1978) John Keats: Complete Poems. Heinemann, London.

[10] Ricks, C. (1976) Keats and Embarrassment. Oxford University Press, Oxford. 